Supporting Information

\title{
Effects of Multiple Pathways on Excited-State Energy Flow in Self-Assembled Wheel-and-Spoke Light-Harvesting Architectures
}

\author{
Hee-eun Song, Christine Kirmaier, Lianhe Yu, \\ David F. Bocian, Jonathan S. Lindsey, and Dewey Holten
}

\section{Contents:}

(1) Titration data for the preparation of the $\mathbf{Z n} \mathbf{n}_{\mathbf{6}} \bullet \mathbf{D P F} \boldsymbol{B O}$ complex.

(2) Transient absorption kinetic data for the $\mathbf{Z} \mathbf{n}_{\mathbf{6}} \bullet \mathbf{D P F b O}$ complex.

(3) Titration data for the preparation of the $\mathbf{Z} \mathbf{n}_{\mathbf{5}} \mathbf{F b \bullet D P F b O}$ complex.

(4) Transient absorption kinetic data for the $\mathbf{Z} \mathbf{n}_{5} \mathbf{F b} \boldsymbol{D P P F O}$ complex.

(5) Titration data for the preparation of the $\mathbf{Z} \mathbf{n}_{4} \mathbf{F} \mathbf{b}_{2} \bullet \mathbf{D P F b O}$ complex.

(6) Transient absorption kinetic data for the $\mathbf{Z} \mathbf{n}_{4} \mathbf{F} \mathbf{b}_{2} \bullet \mathbf{D P F b O}$ complex.

\section{References:}

(1) Li, J.; Ambroise, A.; Yang, S. I.; Diers, J. R.; Seth, J.; Wack, C. R.; Bocian, D. F.; Holten, D.; Lindsey, J. S. J. Am. Chem. Soc. 1999, 121, 8927-8940.

(2) Yu, L.; Lindsey, J. S. J. Org. Chem. 2001, 66, 7402-7419. 

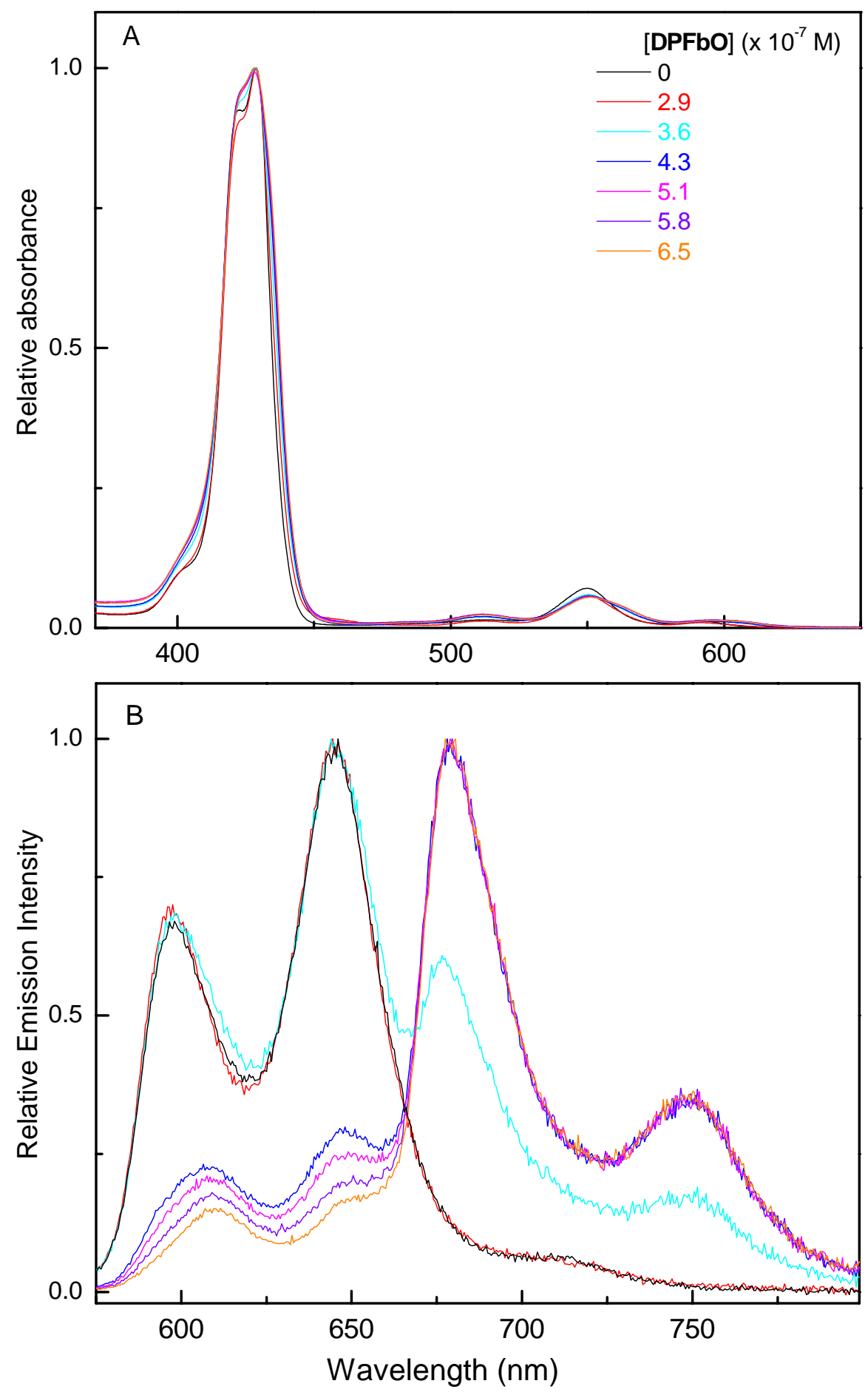

Figure S1. Absorption (A) and emission (B) spectra obtained for the formation of the $\mathbf{Z n}_{\mathbf{6}} \bullet \mathbf{D P F b O}$ host-guest complex upon addition of the DPFbO guest to the $\mathbf{Z} \mathbf{n}_{\mathbf{6}}$ cyclic hexamer host. The concentration of $\mathbf{Z} \mathbf{n}_{\mathbf{6}}$ is $5.1 \times 10^{-7} \mathrm{M}$ for all solutions. Emission spectra were excited at $550 \mathrm{~nm}$. Stock solutions of each component in toluene were prepared using the extinction coefficients for $\mathbf{Z} \mathbf{n}_{\mathbf{6}}$ in toluene (log $\varepsilon=6.43$ at $429 \mathrm{~nm}$ ) from ref 1 , and for DPFbO in toluene ( $\log \varepsilon=5.47$ at $425 \mathrm{~nm}$ ) determined in ref 2 . 


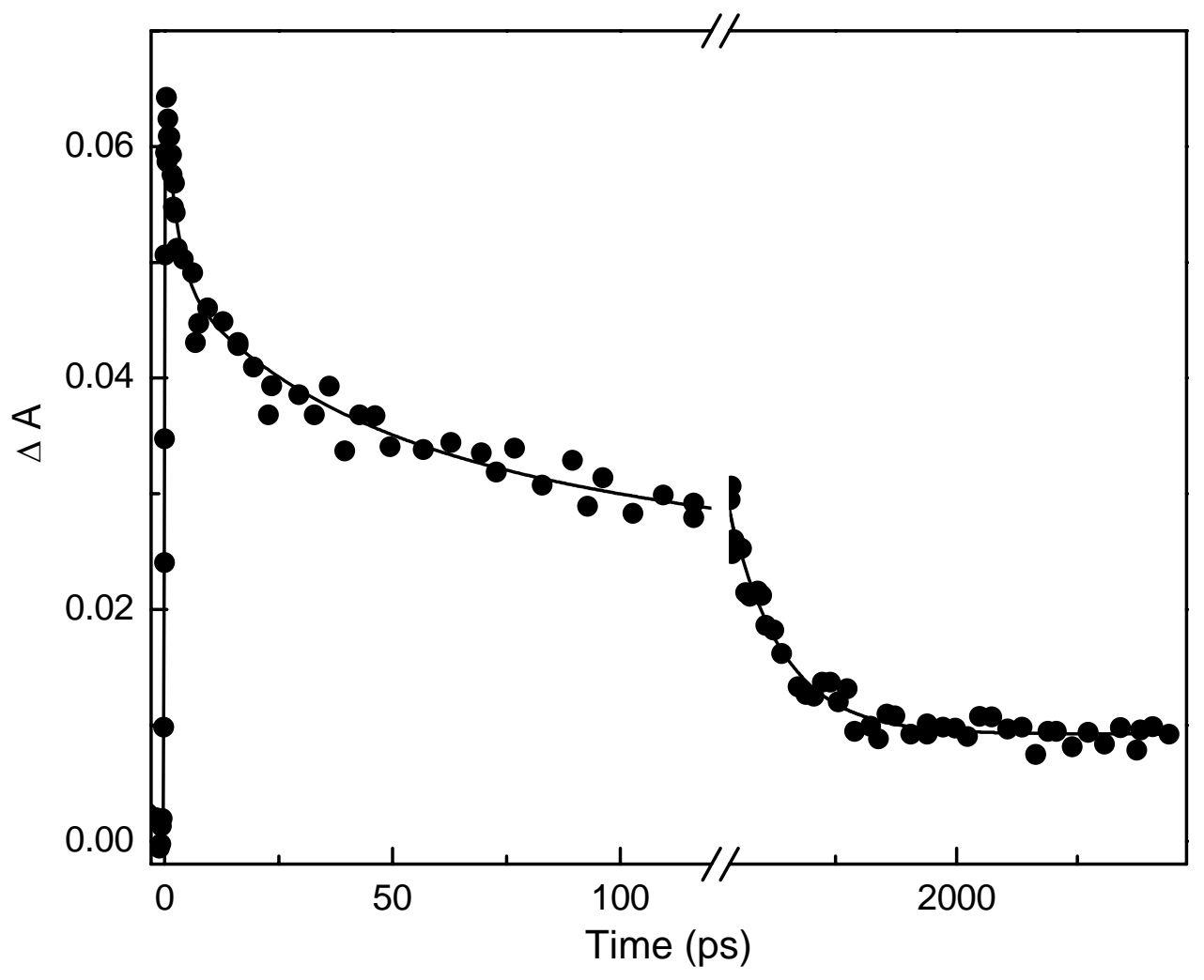

Figure S2. Transient-absorption kinetic data for $\mathbf{Z n}_{\mathbf{6}} \bullet \mathbf{D P F b O}$ in toluene at $685 \mathrm{~nm}$ obtained using excitation with $130 \mathrm{fs}$ flash at $540 \mathrm{~nm}$. The fit is for an instrument response plus three exponentials plus a constant. The average time constants from this wavelength and data spanning 570 to $760 \mathrm{~nm}$ are of $2.7 \pm 0.3 \mathrm{ps}, 40 \pm 10 \mathrm{ps}$, and $540 \pm 70$ ps. 


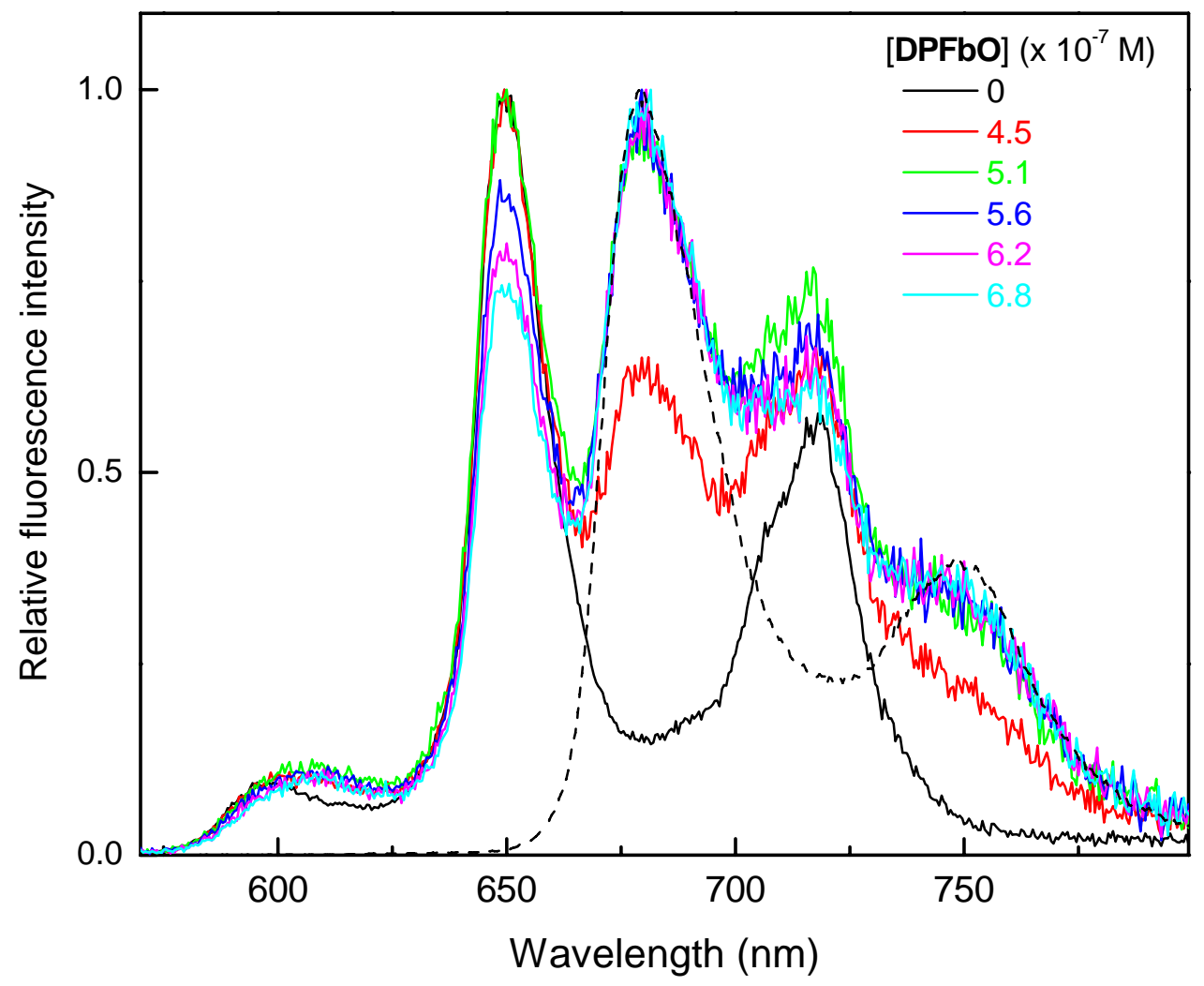

Figure S3. Fluorescence spectra obtained upon formation of the $\mathbf{Z n}_{5} \mathbf{F b} \mathbf{F P F b O}$ host-guest complex upon addition of the $\mathbf{D P F b O}$ guest to the $\mathbf{Z} \mathbf{n}_{5} \mathbf{F b}$ cyclic hexamer host, using excitation at $550 \mathrm{~nm}$. The concentration of $\mathbf{Z n _ { 5 }} \mathbf{F b}$ is $5.7 \times 10^{-7} \mathrm{M}$ for all solutions. The black dashed line is emission spectrum of DPFbO in toluene for comparison. Stock solutions of each component in toluene were prepared using the extinction coefficients for $\mathbf{Z} \mathbf{n}_{5} \mathbf{F b}$ in toluene ( $\log \varepsilon=6.45$ at $428 \mathrm{~nm}$ ) and for DPFbO in toluene ( $\log \varepsilon=5.47$ at $425 \mathrm{~nm}$ ) determined in ref 2. 


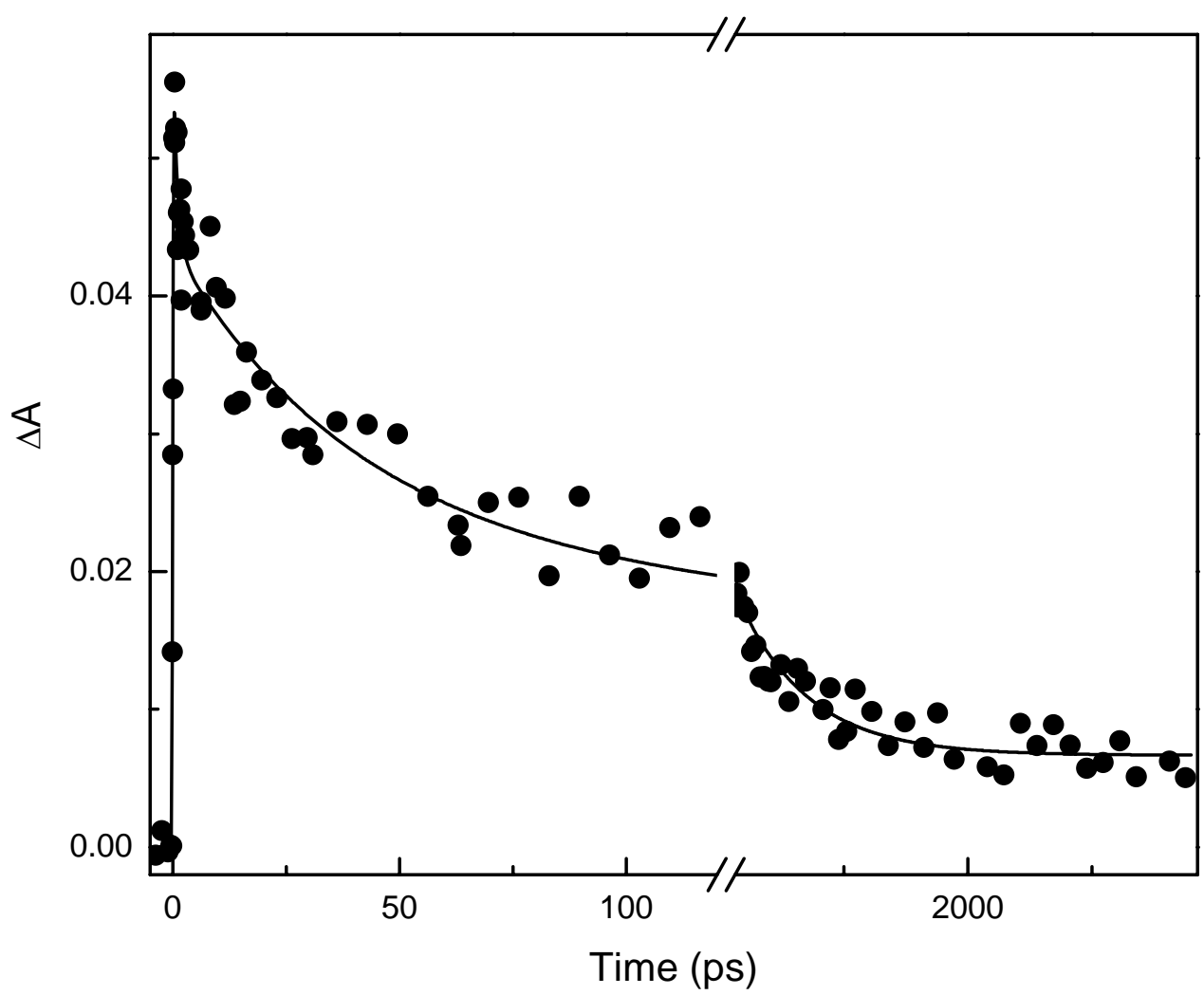

Figure S4. Transient-absorption kinetic data for $\mathbf{Z} \mathbf{n}_{5} \mathbf{F b} \mathbf{D P F b O}$ in toluene at $685 \mathrm{~nm}$ obtained using excitation with $130 \mathrm{fs}$ flash at $540 \mathrm{~nm}$. The fit is for an instrument response plus three exponentials plus a constant. The average time constants from this wavelength and data spanning 570 to $760 \mathrm{~nm}$ are of $3 \pm 1 \mathrm{ps}$, $30 \pm 10$ ps, and $400 \pm 100$ ps. 


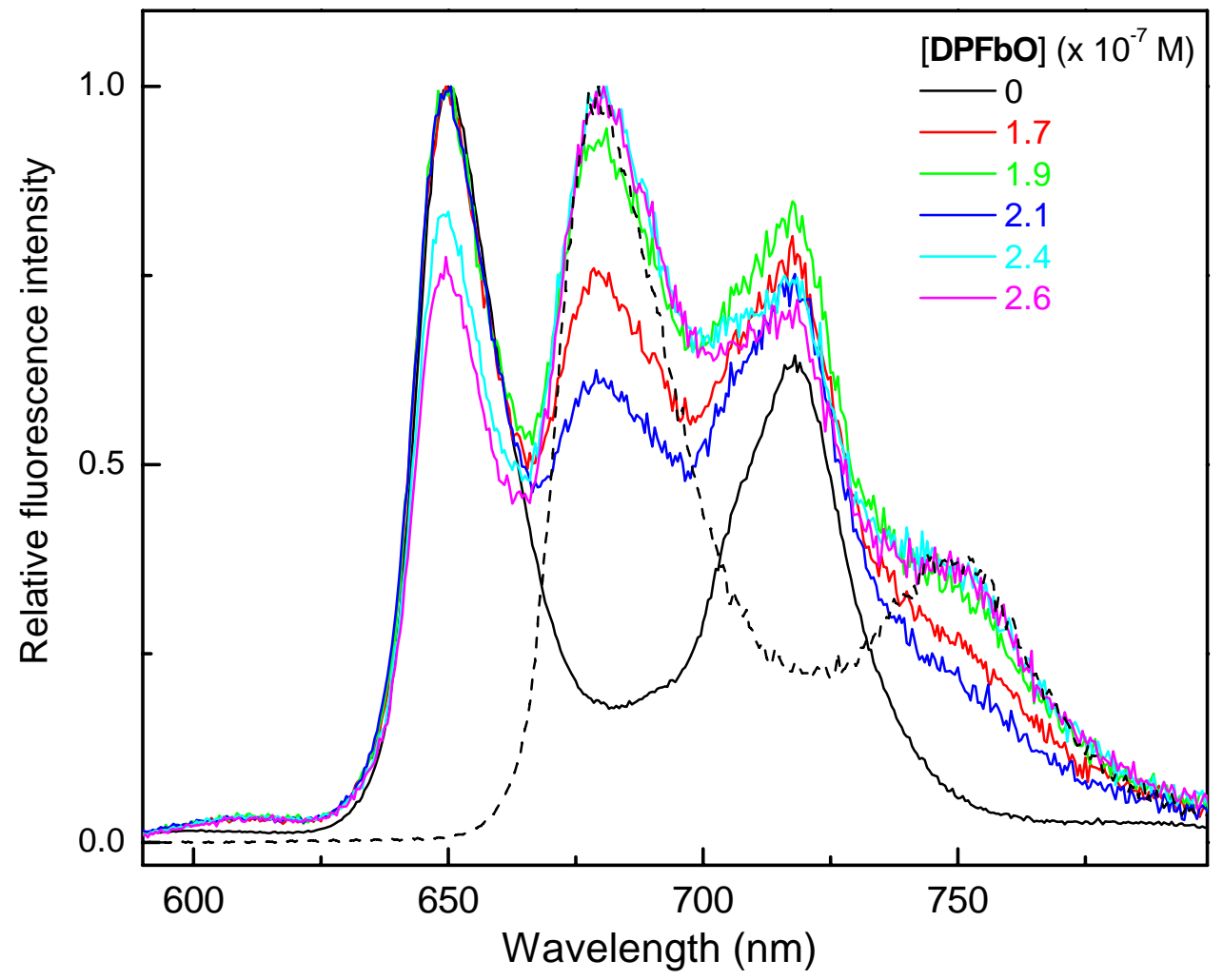

Figure S5. Fluorescence spectra obtained upon formation of the $\mathbf{Z n}_{\mathbf{4}} \mathbf{F} \mathbf{b}_{2} \bullet \mathbf{D P F b O}$ host-guest complex upon addition of the $\mathbf{D P F b O}$ guest to the $\mathbf{Z} \mathbf{n}_{\mathbf{4}} \mathbf{F} \mathbf{b}_{\mathbf{2}}$ cyclic hexamer host, using excitation at $550 \mathrm{~nm}$. The concentration of $\mathbf{Z} \mathbf{n}_{4} \mathbf{F} \mathbf{b}_{2}$ is $2.2 \times$ $10^{-7} \mathrm{M}$ for all solutions. The black dashed line is emission spectrum of DPFbO in toluene for comparison. Stock solutions of each component in toluene were prepared using the extinction coefficients for $\mathbf{Z} \mathbf{n}_{\mathbf{4}} \mathbf{F} \mathbf{b}_{2}$ in toluene (log $\varepsilon=6.37$ at $428 \mathrm{~nm}$ ) and for DPFbO in toluene ( $\log \varepsilon=5.47$ at $425 \mathrm{~nm}$ ) determined in ref 2 . 


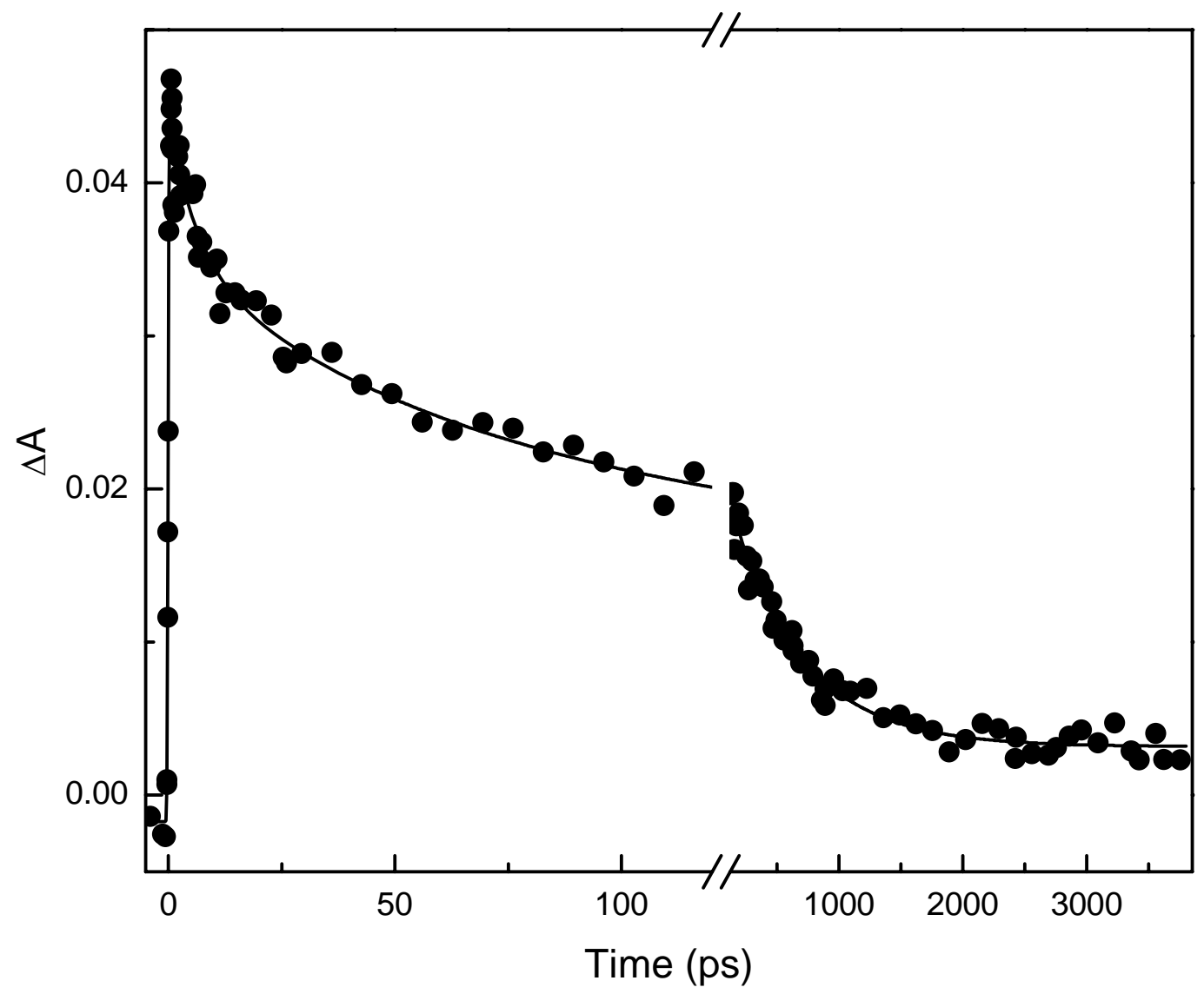

Figure S6. Transient-absorption kinetic data for $\mathbf{Z} \mathbf{n}_{\mathbf{4}} \mathbf{F} \mathbf{b}_{\mathbf{2}} \bullet \mathbf{D P F b O}$ in toluene at 685 nm obtained using excitation with 130 fs flash at $540 \mathrm{~nm}$. The fit is for an instrument response plus two exponentials plus a constant. The average time constants from this wavelength and data spanning 570 to $760 \mathrm{~nm}$ are of $30 \pm 10 \mathrm{ps,}$ and $510 \pm 30$ ps. 